\title{
Are the effects of attention on speed judgments genuinely perceptual?
}

\author{
Matteo Valsecchi, Massimo Vescovi, and Massimo Turatto \\ University of Trento, Rovereto, Italy
}

\begin{abstract}
A number of studies in recent years have suggested that exogenous and endogenous attention might enhance the perceived magnitude of various perceptual attributes, such as contrast and motion speed. Those studies have generally used comparative judgments as a measure to assess the point of subjective equality; however, similarity judgments have been proposed as possibly less prone to decision biases (Schneider \& Komlos, 2008). In three experiments, using a similarity judgment task, we did not find any evidence of motion speed enhancement by exogenous attention. We suggest that the effect revealed by comparative judgments arises at the decisional, rather than the perceptual, stage.
\end{abstract}

A number of studies have been conducted in the last few years with the aim of establishing whether attention alters the way visual stimuli are perceived. In their seminal work, Carrasco, Ling, and Read (2004) first suggested that the perceived contrast of peripheral stimuli increased when attention was exogenously attracted to their location.

Later studies indicated that exogenous attention could increase the perceived intensity of a number of other visual features of static stimuli, such as spatial frequency and gap size (Gobell \& Carrasco, 2005), flicker rate (Montagna \& Carrasco, 2006), and color saturation, but not hue (Fuller \& Carrasco, 2006). Exogenous attention has also been reported to alter the perceived coherence (Liu, Fuller, \& Carrasco, 2006), size (Anton-Erxleben, Henrich, \& Treue, 2007), and speed (Turatto, Vescovi, \& Valsecchi, 2007) of moving objects.

More recent studies have explored different types of attention orienting. Liu, Abrams, and Carrasco (2009) demonstrated that voluntary attention also enhances the perceived contrast of static stimuli. Turatto, Vescovi, and Valsecchi (2008) did not find any evidence of an increase in perceived speed when visual attention was oriented by stimuli held in working memory.

All of the aforementioned studies used adaptations of the experimental paradigm introduced by Carrasco et al. (2004) - that is, a $2 \times 2$ alternative forced choice (AFC) task with simultaneous stimulus presentation. In the original task, the participant was presented with two simultaneous Gabor patches and was asked to press one of four keys to report the orientation (index vs. ring finger) of the highest contrast (left vs. right hand) Gabor. Task instructions stressed the relevance of the orientation question in order to minimize possible cue-congruent response biases on the contrast question. Attention was summoned by a black dot that appeared above the position of either the right or the left Gabor or above the fixation point (the latter case served as a control condition). This exogenous cue was presented $120 \mathrm{msec}$ prior to the appearance of the Gabors, a stimulus onset asynchrony (SOA) that is well suited for transient attention to be effective.

A number of control experiments have been devised in order to avoid two possible confounds that are inherent in this experimental procedure. First, one may ask whether the effects observed in this paradigm are genuinely attentional. Control experiments in Gobell and Carrasco (2005; see also Anton-Erxleben et al., 2007), in which judgments were unaffected by a postcuing procedure, and in the original article by Carrasco et al. (2004; see also Liu et al., 2006; Turatto et al., 2007), in which judgments were likewise unaffected by cues presented $500 \mathrm{msec}$ before target onset, seem to indicate that the effect of exogenous cues on the way visual attributes are perceived is indeed attentional. Second, Schneider (2006) proposed that the effect of cuing on perceived contrast could be due to direct sensory interaction between cue and probe stimuli, rather than to the orienting of attention. In particular, Schneider observed that the effect of peripheral cues on the perceived brightness contrast of filled dots presented at the same location crucially depended on the luminance polarity of the cue; namely, white cues increased perceived contrast, but black cues tended to reduce it. However, Ling and Carrasco (2007) in their reply to Schneider, demonstrated that both white and black cues increased the perceived Michelson contrast of Gabor patches, showing that the point of subjective equality (PSE) change reported by Carrasco et al. (2004) was produced by visual attention, and not by the sensory interaction between the cue and probe stimuli.

Once it is established that the results obtained using peripheral cues and $2 \times 2 \mathrm{AFC}$ comparative judgments are

M.Valsecchi, matteo.valsecchi@gmail.com 
indeed genuinely attentional, the second question one has to deal with is whether the effects of attention occur at the perceptual level. In order to exclude any simple response biases, Carrasco et al. (2004) asked participants to use either the left or the right hand to indicate which of two simultaneously presented Gabors had the lower contrast, as opposed to indicating which one had the higher contrast. There was a reversal of the effect of cuing that occurred together with the reversal of the question, thus ruling out the hypothesis that the participants had a straightforward tendency to press the key corresponding to the cued stimulus. Similar control experiments with similar results have been reported in a number of studies (e.g., AntonErxleben et al., 2007; Gobell \& Carrasco, 2005; Turatto et al., 2007). These findings excluded the possibility of some less straightforward response biases as well-for example, the one that might have emerged if the participants had chosen to report the orientation or the direction of motion of the cued stimulus because the task was made easier by attention.

A further hypothesis, which involves a nonperceptual interpretation of Carrasco et al.'s (2004) results, has been suggested by Prinzmetal, Long, and Leonhardt (2008). The authors suggested that, when near-threshold stimuli are used, participants may guess that a stimulus had been presented near the cued location and, thus, may infer and report that it had a higher contrast. This account, however, has been rejected, given that Carrasco, Fuller, and Ling (2008) showed that the cues used by Carrasco et al. (2004) were clearly suprathreshold, while further replicating the previous attentional modulation.

Finally, a nonperceptual account of the effect of spatial cuing on judgments of contrast intensity was recently introduced by Schneider and Komlos (2008). In particular, the authors suggested that the effect of spatial attention on judgments of contrast intensity is the consequence of the prioritization of the cued information, which biases the decision process. Schneider and Komlos distinguished between a perceptual stage, a decision stage, and a subsequent response production stage. Although excluding the possibility that the cuing effect emerges at the response production stage, the reversal of the cuing effect observed after the reversal of the question (e.g., Carrasco et al., 2004) does not imply that the effect is perceptual in nature, since it may take place at the decision level. What Schneider and Komlos suggested is that participants might make their decision on the basis of an absolute scale and, subsequently, provide a response corresponding to the lower contrast Gabor, making that manipulation ineffective in excluding decision biases. In particular, when two stimuli appear to be identical along the dimension of interest and one is prioritized by attention (i.e., made more salient or more easily selectable), participants might assume that the attended stimulus is superior to the unattended one (along the dimension of interest) and, thus, respond coherently. The reverse-instruction control experiments rest on the assumption that response generation and perceptual decision are the same process. If one assumes that they are two distinct processes, decision and response biases might be independent. The authors used similarity judg- ments as a way to circumvent possible attention-induced decision biases. When participants are asked to perform a similarity judgment, they cannot decide in favor of the cued stimulus when uncertain, as in the case of higher/ lower judgments. In principle, in this case, merely shifting the criterion should affect only the total number of same responses, leaving the central tendency of the curve unchanged.

In two experiments, Schneider and Komlos (2008) replicated the findings in Carrasco et al. (2004) with comparative contrast judgments, but when similarity judgments were used (with the same stimuli), the participants' responses did not show any sign of a cuing effect. The authors concluded that the effect of exogenous cues on forced choices about apparent contrast emerges at the decision stage and is not genuinely perceptual.

Given Schneider and Komlos's (2008) demonstration of decision biases, rather than modulation of perceived contrast, the question arises as to whether the reported modulation of other perceptual attributes is also due to decision biases. First of all, it should be noted that there are examples of effects produced by exogenous cues that are genuinely perceptual-specifically, those in which exogenous cues have been shown to reduce the intensity of a visual attribute. In order for attention to produce a decision bias independently from the way the question is asked, an intrinsic magnitude must be defined for the visual attribute, and if attention has the effect of causing participants to decide in favor of the attended stimulus, a decision bias can produce only an increase of the estimated PSE value. One case is the reduction of perceived brightness contrast induced by black cues in Schneider (2006), and another example comes from Experiment 4 in Turatto et al. (2007), where we found that peripheral cues reduced the perceived contrast of moving stimuli. In principle, the change in perceived speed that we found in our previous studies could be a genuinely perceptual effect, and the only way to test whether this is the case is to try to replicate the finding of Turatto et al. (2007) using similarity judgments.

In the first three experiments of the present study, we assessed the effect of the cuing procedure used in Turatto et al. (2007) on PSEs estimated from similarity judgments performed in isolation or combined with comparative judgments. To anticipate the results, we failed to find any effect of cuing in similarity judgments. This result cannot be attributed to any methodological flaw in our procedure, since in a fourth control experiment, we confirmed that our similarity judgment procedure was capable of detecting physical speed differences within the order of magnitude of the purported attentional effects revealed by comparative judgments.

\section{EXPERIMENT 1}

In Experiment 1, we asked whether both similarity and comparative judgments would reveal an increase in the perceived speed of exogenously cued moving Gabor patches, using stimuli and a procedure similar to the ones introduced by Turatto et al. (2007; see also Turatto et al., 
2008). In order to make sure that both kinds of judgments shared the same perceptual stage, on each trial, we asked the participants to report which of the two Gabor patches moved faster (encouraging them to guess if they had not noticed any difference) and, subsequently, to report whether they had perceived the two stimuli as moving at different speeds.

\section{Method}

Participants. Fourteen students from the University of Trento (mean age, 23.6 years; 8 of them female) volunteered for participation in exchange for course credits or $€ 6$. All of the participants were naive as to the aim of the study and reported normal or correctedto-normal visual acuity. All of the participants gave their informed consent, and the experiment was conducted in accordance with the Declaration of Helsinki.

Stimuli and Procedure. The stimuli were presented on a 19-in. Iiyama CRT monitor $(1,024 \times 768$ pixels, $150 \mathrm{~Hz})$. The stimuli were generated and the experiment was controlled using MATLAB and the Psychophysics Toolbox (Pelli, 1997), running under Windows XP on a Pentium IV Dell PC. Viewing distance was $60 \mathrm{~cm}$.

The stimuli were presented on a gray background $\left(27.7 \mathrm{~cd} / \mathrm{m}^{2}\right)$. Each trial began with the onset of a black fixation point $\left(0.2^{\circ}\right.$ of visual angle in diameter) in the center of the screen, together with two black circular placeholders ( $3^{\circ}$ in diameter) $5^{\circ}$ to the right and the left of the fixation point (see Figure 1). The exogenous cue, a black disk $\left(0.6^{\circ}\right.$ in diameter), was flashed $500 \mathrm{msec}$ later for $60 \mathrm{msec}, 2^{\circ}$ above the right or left placeholder. After another 80 -msec interstimulus interval, two moving Gabor patches (vertical sinusoidal achromatic gratings enveloped by a Gaussian filter; $3^{\circ} \times 3^{\circ}, 60 \%$ contrast, 1 -cpd spatial frequency) were presented inside the placeholders. The duration of the two Gabors was $200 \mathrm{msec}$, and they drifted independently to the right or the left. On each trial, one of the two Gabors (standard), located randomly on the left or the right side, moved at a fixed speed of $4.3^{\circ} / \mathrm{sec}$, whereas the speed of the other Gabor (test) was chosen from a possible set of seven roughly linearly spaced levels $(1.3,2.3$, $3.3,4.3,5.4,6.3,7.2) .{ }^{1}$ Immediately after the two moving Gabors, a question mark flanked by two arrows appeared, which prompted the participants to indicate which of the two Gabors moved faster by

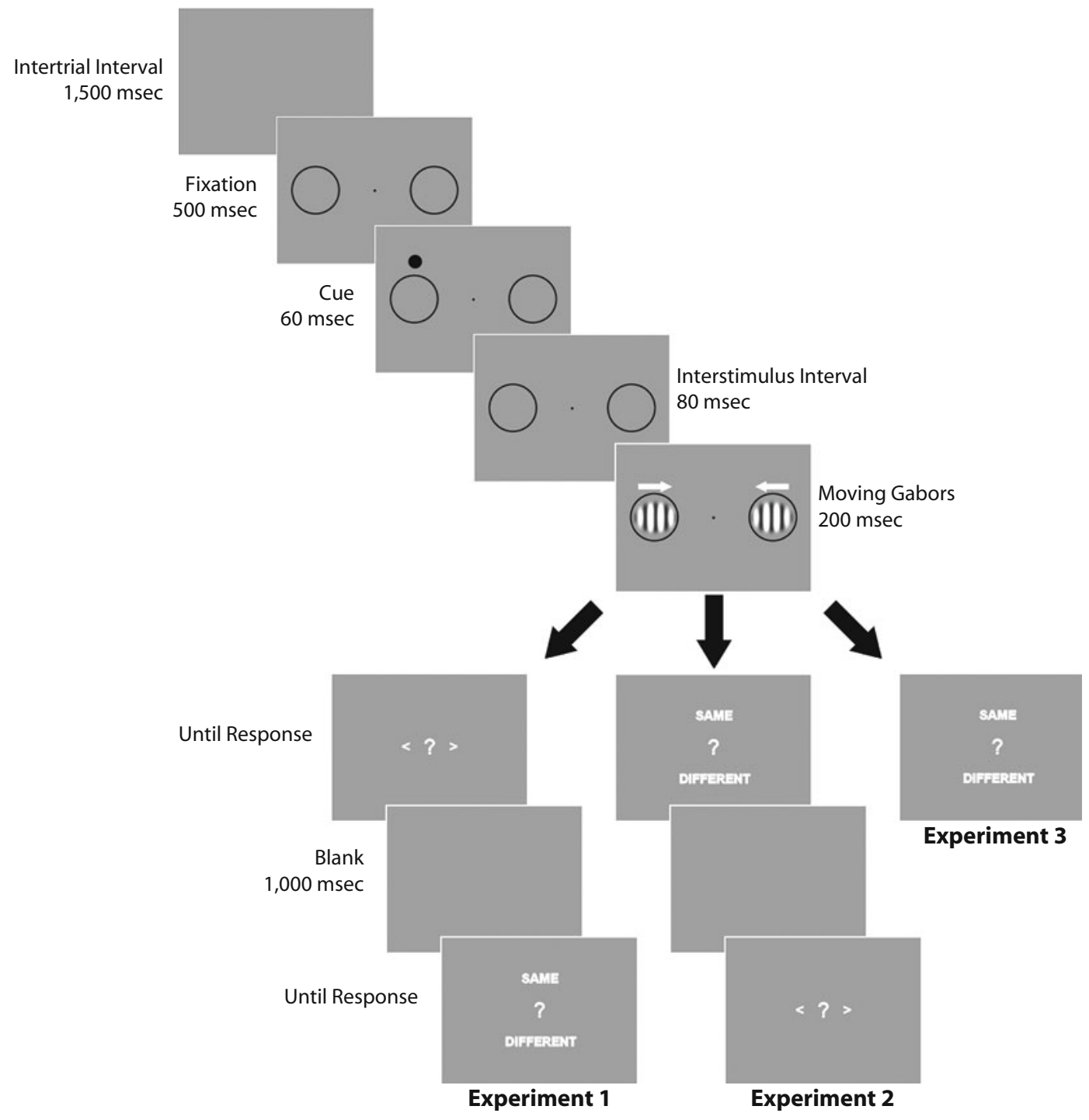

Figure 1. Trial procedure in Experiments 1 (left), 2 (center), and 3 (right). Note that the trial sequence is identical in all three experiments up to the presentation of the moving Gabors. The white horizontal arrows indicate the direction of movement and were not actually presented during the experiments. 
pressing either the left arrow (left Gabor) or the right arrow (right Gabor) on the keyboard. After the response, a blank screen was presented for 1,000 msec, followed by another question mark and the words "Same" and "Different" above and below the question mark, respectively. At this point, the participants were to press either the up arrow key, to indicate that they had perceived the stimuli as moving at the same speed, or the down arrow key, to indicate that they moved at different speeds. The mapping of "Same" and "Different" responses to up and down arrow keys was alternated between participants. A final blank lasting $1,500 \mathrm{msec}$ was presented between the second response and the following trial.

The participants were encouraged to respond "Different" if they had noticed a difference between the speed of the two moving Gabors. Moreover, they were encouraged to guess which of the two Gabors was moving faster when they had not noticed any difference.

Design. Each participant underwent 592 trials overall. A $2 \times 7$ factorial design was used, with condition (test cued vs. standard cued) and test speed (see above) as factors and with 40 trials in each cell of the design. The experiment was divided into five blocks of 112 trials each, plus 32 practice trials at the beginning of the experiment. The experiment lasted approximately $50 \mathrm{~min}$.

Data analysis. A cumulative Gaussian curve

$$
\Phi\left(\frac{x-\mu}{\sigma}\right)
$$

and a Gaussian curve

$$
\frac{a}{\sigma} \varphi\left(\frac{x-\mu}{\sigma}\right)
$$

were fit to the comparative judgment and to the similarity judgment data for each participant, respectively.

The participants were discarded from the analysis if the amplitude of the $95 \%$ confidence interval for the mean parameter $(\mu)$ of the function fit to either the comparative or the similarity judgment data exceeded $3.5^{\circ} / \mathrm{sec}$ in either the test-cued or the standard-cued condition, indicating a poor fit.

Paired $t$ tests were conducted on estimated PSEs (i.e., the $\mu$ parameter of the fitted functions) in order to reveal possible differences in the perceived speed between the two conditions (standard cued vs. test cued). The same analysis was conducted on both the PSE measure obtained from the comparative judgment task and the PSE measure obtained from the similarity judgment task. Furthermore, we performed the same tests on the remaining parameters ( $\sigma$ and $a$, the latter only in the case of similarity judgments) in order to check whether the effect of cuing was limited to the offset of the psychometric curves or whether it influenced their shape as well.

\section{Results and Discussion}

Three participants were discarded from the analysis. All 3 had a poor fit to at least one comparative judgment curve, and 1 of them also had a poor fit to one of the similarity judgment curves. The average $R^{2}$ of the single participant fits was .97 in the case of the cumulative Gaussian curves and .90 in the case of the Gaussian curves.

The results of the comparative judgments are depicted in Figure 2A. The curve for the test-cued condition was clearly shifted to the left, as compared with the one for the standard-cued condition, indicating that the speed of the standard Gabor was matched to smaller values of the test Gabor when the latter was cued and vice versa. The mean value of the PSE for the test-cued condition was $4.17^{\circ} / \mathrm{sec} \pm 0.10$ (between-participants $S E M$ ), whereas it was $4.71 \% \mathrm{sec} \pm 0.11$ for the standard-cued condition (Figure 3). The difference between the two PSE values, as shown by the paired $t$ test, was significant $[t(10)=2.77$, $p<.019$ ].

The mean value of $\sigma$ for the test-cued condition was $1.65^{\circ} / \mathrm{sec} \pm 0.39$, whereas it was $1.95^{\circ} / \mathrm{sec} \pm 0.38$ for the standard-cued condition. The difference between the two PSE values, as shown by the paired $t$ test, was significant $[t(10)=2.95, p<.014]$. Individual values of PSE and $\sigma$ are depicted in Figure 3. A significant (positive) linear relationship between $\sigma$ and PSE was evident only in the standard-cued condition.

The results for the similarity judgments are depicted in Figure 2B. In this case, the curves almost perfectly over-

\section{A}

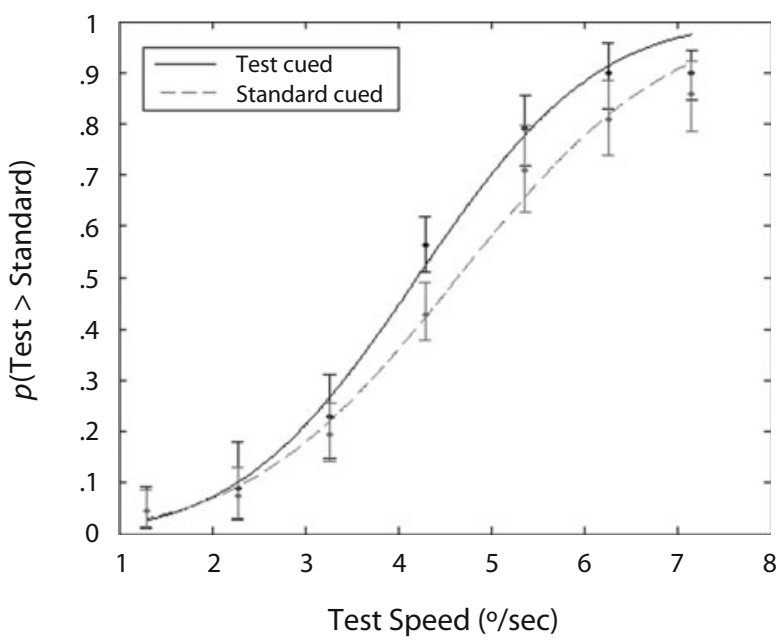

B

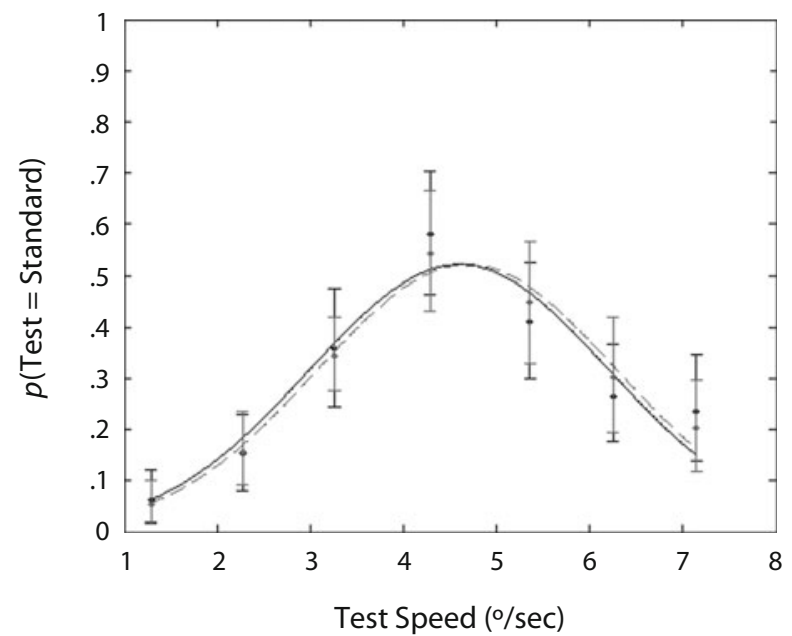

Figure 2. Comparative judgment (A) and similarity judgment (B) results in Experiment 1 for the standard-cued and test-cued conditions. A cumulative Gaussian curve (A) and a Gaussian curve (B) were fitted to the mean data for the comparative judgment and the similarity judgment measures, respectively. Error bars indicate $95 \%$ of the distribution of the mean computed from 10,000 bootstrap samples obtained by random resampling with replacement. 
Test Cued
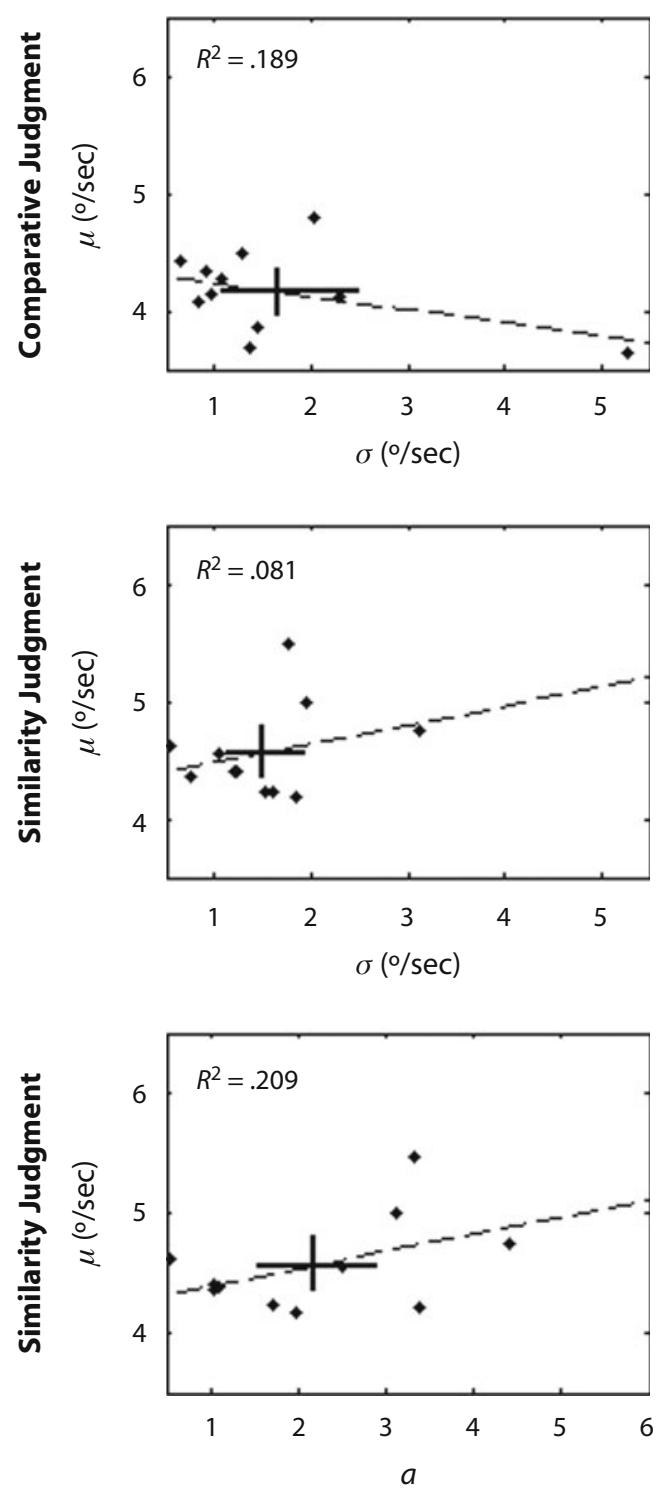

Standard Cued
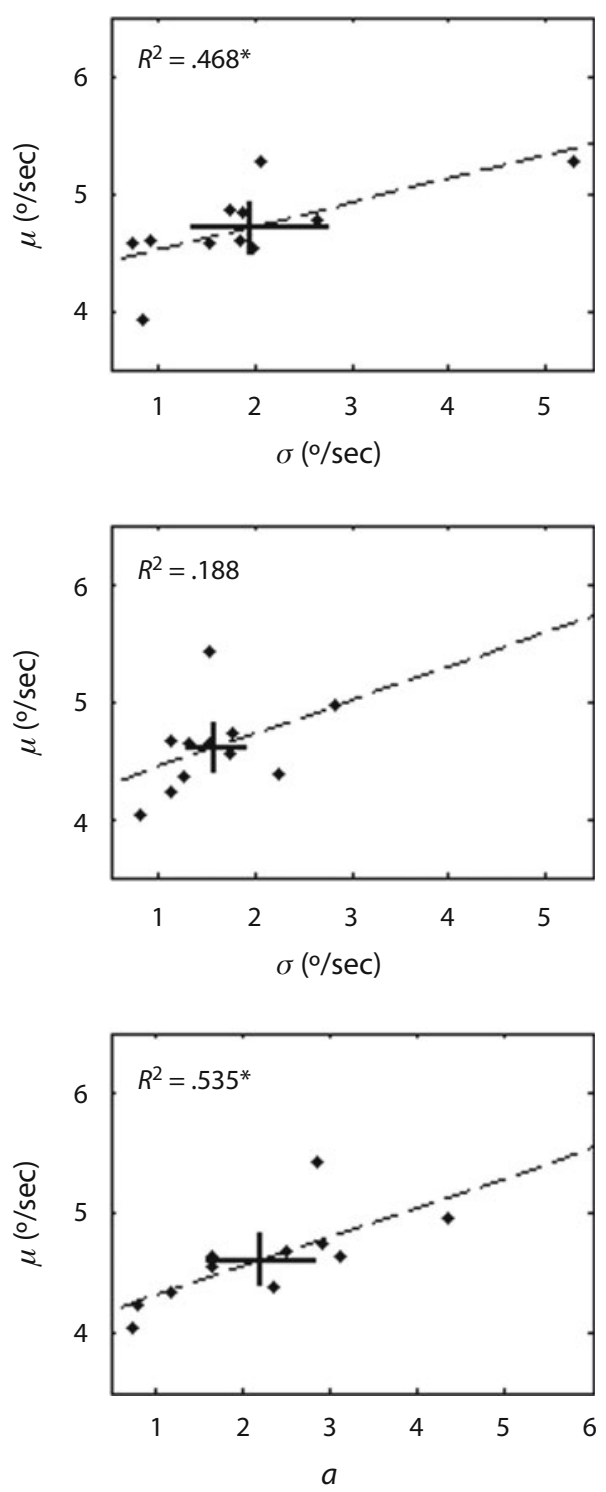

Figure 3. Relationship between fit parameters in Experiment 1. Each point represents a participant and a given condition. The dashed line represents the regression line; the $R^{2}$ value is relative to the linear regression between the two variables. Asterisks indicate a significant $(p<.05)$ linear relationship. Error bars indicate $95 \%$ of the distribution of the mean computed from 10,000 bootstrap samples obtained by random resampling with replacement. The coordinates of the crossing point of the two error bars are the between-participants means of the two parameters.

lapped. The mean value of the PSE for the test-cued condition was $4.56^{\circ} / \mathrm{sec} \pm 0.12$, whereas it was $4.60^{\circ} / \mathrm{sec} \pm$ 0.12 for the standard-cued condition. The difference between the two PSE values was not significant $[t(10)=$ $0.35, p=.732]$.

Cuing did not have a significant effect on the $\sigma$ and $a$ parameters either (both $t \mathrm{~s}<1$, both $p \mathrm{~s}>.466$ ). Individual values of PSE, $\sigma$, and $a$ are depicted in Figure 3 . The only significant (positive) linear relationship was observed between $a$ and PSE in the standard-cued condition.
The results of the comparative judgments fully replicated the results previously reported by Turatto et al. (2007, 2008). That is, cued stimuli were matched to faster moving stimuli, a result compatible with the hypothesis that exogenous attention enhances the perceived speed of moving stimuli. However, the results of the similarity judgments, which were obtained from the same participants and for the same stimuli, seem to disconfirm this conclusion, given the absence of any difference between the two functions. 
Since it is reasonable to assume that both judgments share the same perceptual stage, the attentional effect that emerged in the comparative judgments is bound to happen at a later stage and to be decisional in nature. A possible confound has, however, to be ruled out before this conclusion is accepted. In Experiment 1, the similarity judgment was always performed after the comparative judgment. The delay between the stimulus presentation and the intervening comparative judgment might have degraded the trace of the perceived speed, canceling out the effect of the attentional manipulation when measured through similarity judgments. This confound was addressed in Experiment 2.

\section{EXPERIMENT 2}

In Experiment 2, we asked participants first to perform the similarity judgment and, subsequently, to perform the comparative judgment. If the lack of any attentional effect for the similarity judgment in Experiment 1 was due to the degradation of the perceptual representation over time, an opposite pattern of results (i.e., a cuing effect on the similarity measure and possibly no cuing effect on the comparative judgment) should be observed when the order of the tasks was reversed.

\section{Method}

Participants. Ten students from the University of Trento (mean age, 21.6 years; 6 of them female) volunteered for participation in Experiment 2 in exchange for course credits or $€ 6$. All of the participants were naive as to the aim of the study and reported normal or corrected-to-normal visual acuity. None of the participants had taken part in Experiment 1. All of the participants gave their informed consent, and the experiment was conducted in accordance with the Declaration of Helsinki.

Stimuli and Procedure. The stimuli and procedure were the same as those in Experiment 1, with the exception that the partici- pants were asked to perform the similarity judgment first and, subsequently, to provide the comparative judgment (see Figure 1).

Design and Data analysis. The design and data analysis were the same as those in Experiment 1.

\section{Results and Discussion}

One participant was discarded from the analysis because he had a poor fit to at least one comparative judgment curve. The average $R^{2}$ of the single participant fits was .96 in the case of the cumulative Gaussian curves and .87 in the case of the Gaussian curves.

The results of the comparative judgments are depicted in Figure 4A. The curve for the test-cued condition was clearly shifted to the left, as compared with the one for the standard-cued condition. The mean value of the PSE for the test-cued condition was $4.05^{\circ} / \mathrm{sec} \pm 0.12$, whereas it was $4.74 \% \mathrm{sec} \pm 0.14$ for the standard-cued condition. The difference between the two PSE values, as shown by the paired $t$ test, was significant $[t(8)=2.81, p<$ .022].

The value of $\sigma$ for the test-cued condition was $1.77^{\circ} / \mathrm{sec} \pm 0.26$, whereas it was $2.03^{\circ} / \mathrm{sec} \pm 0.31$ for the standard-cued condition. The difference between the two $\sigma$ values, as shown by the paired $t$ test, failed to reach significance $[t(8)=2.24, p=.056]$. Individual values of PSE and $\sigma$ are depicted in Figure 5. No significant linear relationship between $\sigma$ and PSE was observed.

The results of the similarity judgments are depicted in Figure 4B. The mean value of the PSE for the testcued condition was $4.62^{\circ} / \mathrm{sec} \pm 0.19$, whereas it was $4.53^{\circ} / \mathrm{sec} \pm 0.07$ for the standard-cued condition. The difference between the two PSE values was not significant $[t(8)=0.50, p=.629]$.

Cuing did not have a significant effect on the $\sigma$ and $a$ parameters either (both $t \mathrm{~s}<1$, both $p \mathrm{~s}>$.393). Individual values of PSE, $\sigma$, and $a$ are depicted in Figure 3.
A

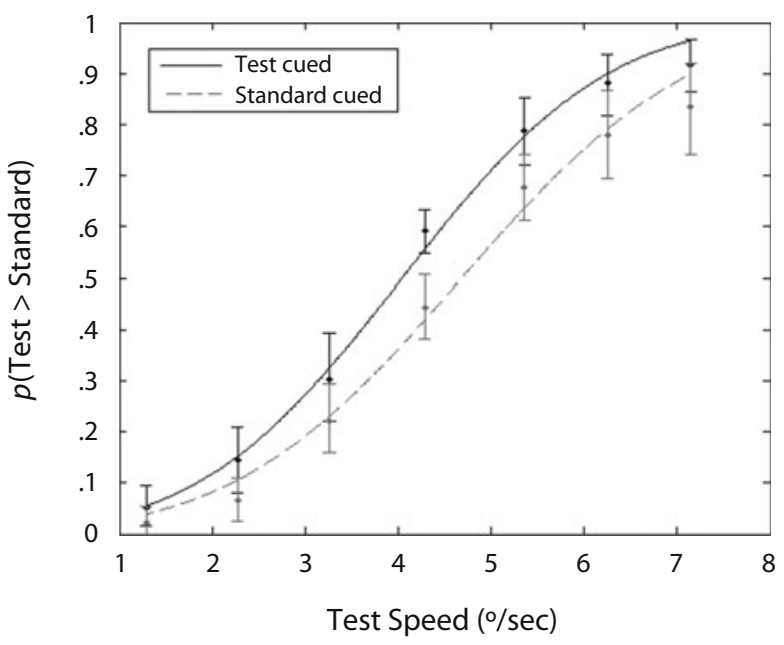

B

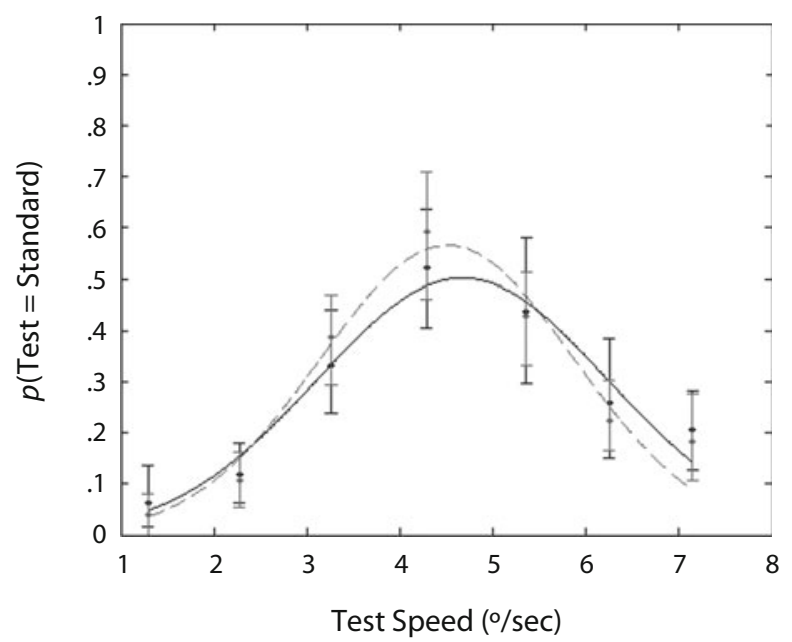

Figure 4. Comparative judgment (A) and similarity judgment (B) results in Experiment 2 for the standard-cued and test-cued conditions. A cumulative Gaussian curve (A) and a Gaussian curve (B) were fitted to the mean data from the comparative and the similarity measures, respectively. Error bars indicate $95 \%$ of the distribution of the mean computed from 10,000 bootstrap samples obtained by random resampling with replacement. 
Test Cued
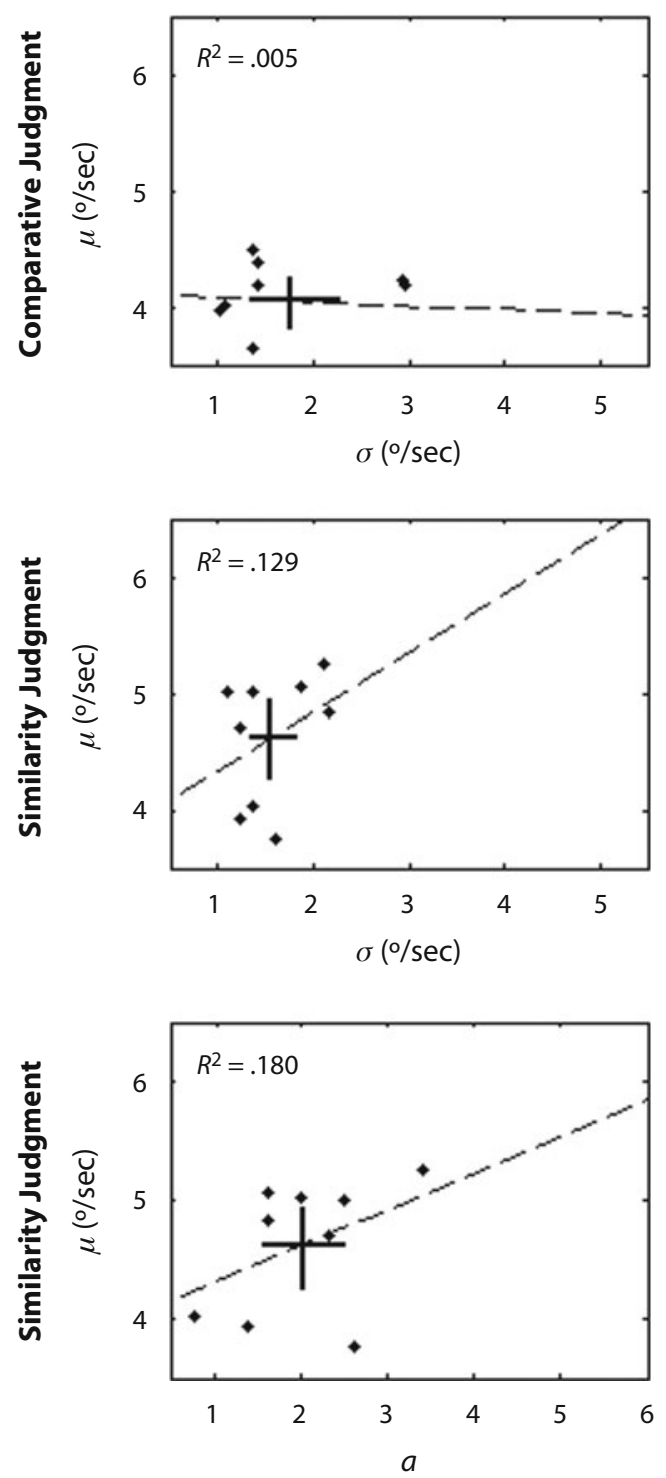

Standard Cued
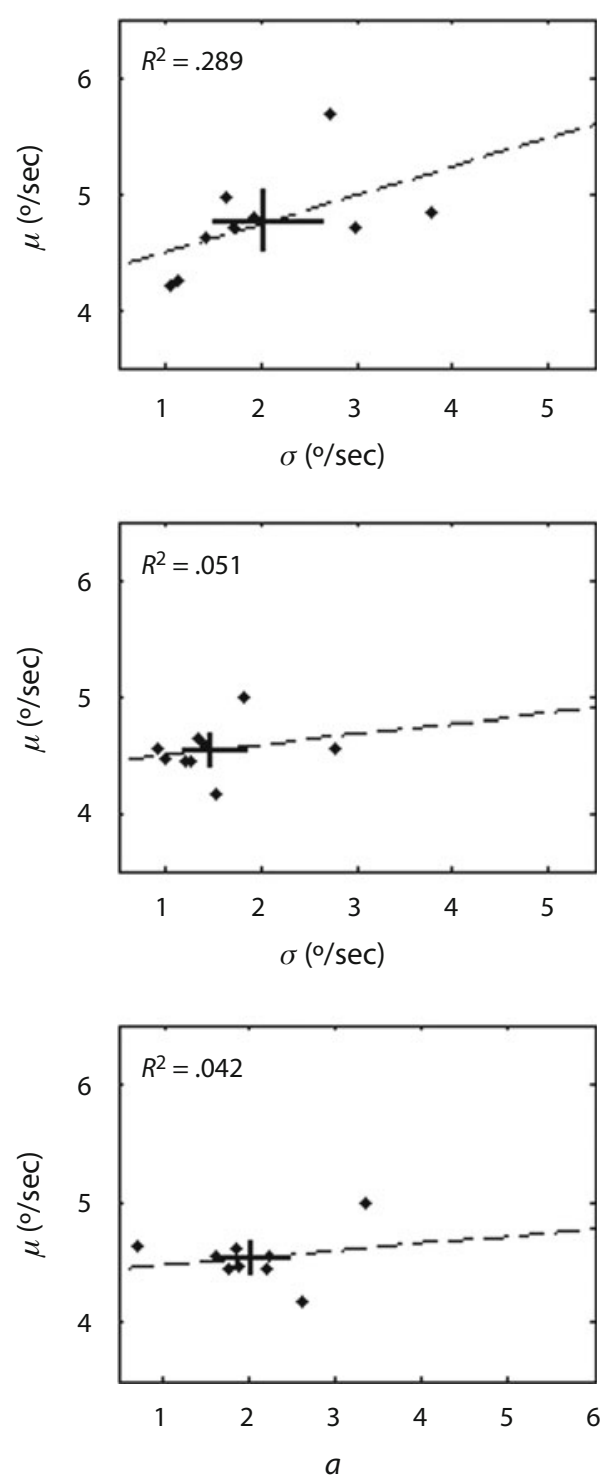

Figure 5. Relationship between fit parameters in Experiment 2. Each point represents a participant and a given condition. The dashed line represents the regression line; the $R^{2}$ value is relative to the linear regression between the two variables. No significant linear relationship was observed in any condition. Error bars indicate $95 \%$ of the distribution of the mean computed from 10,000 bootstrap samples obtained by random resampling with replacement. The coordinates of the crossing point of the two error bars are the between-participants means of the two parameters.

No significant linear relationship was observed between $\sigma, a$, and the PSE in any condition.

Despite being collected after the similarity judgments, in Experiment 2, the comparative judgments provided the same reliable cuing effect as that in Experiment 1; that is, cued stimuli were consistently matched to faster stimuli. On the contrary, no reliable cuing effect was observed for the similarity judgment, thus fully replicating the results of Experiment 1.

The fact that in Experiment 1, attention affected only comparative judgments, without producing a reliable ef- fect on similarity judgments, was not due to a possible degradation of the perceptual trace during the time interval between stimulus presentation and judgment. Indeed, the same pattern of results was observed in Experiment 2, where the order of the tasks was reversed.

However, since the participants performed two judgments on the same trial, it is still possible that this might have generated some interference between the two decisions. In order to exclude this last possibility, in Experiment 3 , we asked the participants to perform only the similarity judgment. 


\section{EXPERIMENT 3}

As was noted by Schneider and Komlos (2008), when participants perform the two judgment tasks in sequence, they are prone to prioritizing one of the two tasks, which might reduce the reliability of the results in the nonprioritized task. In order to exclude this possibility, in the present experiment, we asked the participants to perform only the similarity judgment task. We have already demonstrated, in a number of experiments, that if participants are asked to perform comparative judgments in a similar paradigm (Turatto et al., 2007, 2008), they reliably indicate the cued Gabor as moving faster. The decisional account of the cuing effect on comparative judgments would, however, predict that, in the same condition, exogenous cues should not affect similarity judgments.

\section{Method}

Participants. Eleven students from the University of Trento (mean age, 21.5 years; 7 of them female) volunteered for participation in Experiment 3 in exchange for course credits or $€ 6$. All of the participants were naive as to the aim of the study and reported normal or corrected-to-normal visual acuity. None of the participants had taken part in Experiment 1 or 2. All of the participants gave their informed consent, and the experiment was conducted in accordance with the Declaration of Helsinki.

Stimuli and Procedure. The stimuli and procedure were the same as those in Experiment 2, with the exception that the trial terminated after the participants provided the similarity judgment (see Figure 1).

Design and Data analysis. The design and data analysis were the same as those in Experiments 1 and 2 as far as the similarity judgment task was concerned.

\section{Results and Discussion}

Two participants were discarded from the analysis because they had a poor fit to at least one curve. The average $R^{2}$ of the single participant fits was .92 .

The results of Experiment 3 (similarity judgments) are depicted in Figure 6. The mean value of the PSE for the test-cued condition was $4.84^{\circ} / \mathrm{sec} \pm 0.17$, whereas it was $5.01 \%$ sec \pm 0.13 for the standard-cued condition (Figure 7). The difference between the two PSE values was not significant $[t(8)=0.69, p=.506]$.

Cuing did not have a significant effect on the $\sigma$ and $a$ parameters either (both $t \mathrm{~s}<1$, both $p \mathrm{~s}>.774$ ). Individual values of PSE, $\sigma$, and $a$ are depicted in Figure 7. No significant linear relationship was observed between $\sigma, a$, and the PSE in any condition.

The data showed no sign of attentional effects when similarity judgments were used. The present result cannot be attributed to a dual-task interference from the concomitant comparative judgment task, as might have been the case in Experiments 1 and 2. This suggests that the effect of exogenous cues on perceived speed likely does not emerge at the perceptual stage.

It is worth noting that the overall frequency of same responses was numerically higher than in the previous two experiments. This might be due to a general bias that reduced the tendency of the participants to judge the two stimuli as same while being forced to choose one of the

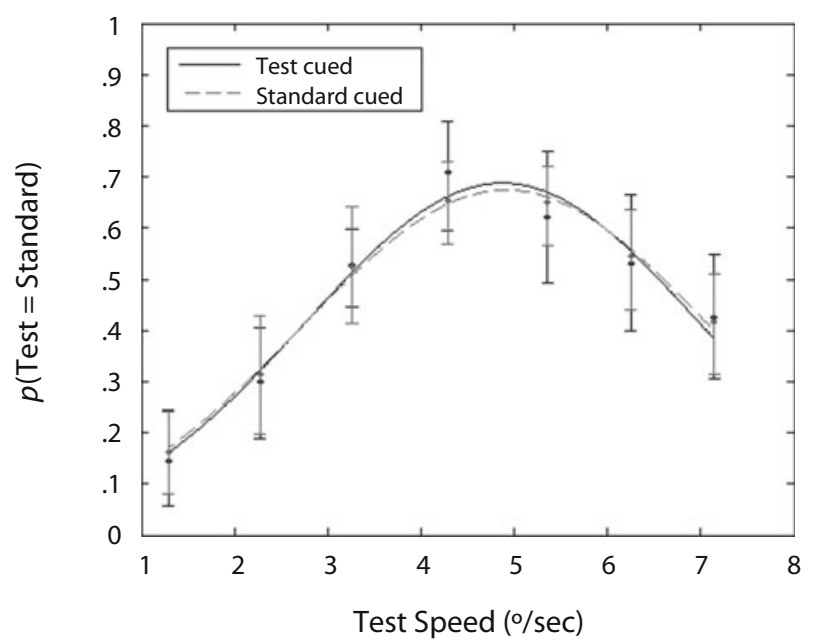

Figure 6. Similarity judgment results in Experiment 3 for the standard-cued and test-cued conditions. A Gaussian curve was fitted to the mean data. Error bars indicate $95 \%$ of the distribution of the mean computed from 10,000 bootstrap samples obtained by random resampling with replacement.

two as faster. Given that the PSE is estimated only by the mean of the fitted distribution, independently of the overall response rate, this bias does not affect our main conclusion.

Overall, the results of the first three experiments indicate that exogenous attention does not change the PSEs when estimated by means of similarity judgments. However, before drawing further conclusions, it has to be demonstrated that the similarity judgment task is sensitive enough to provide different PSE values when different values of speed reach the decision stage. This was tested in Experiment 4.

\section{EXPERIMENT 4}

The lack of any effect of cuing on the PSE values obtained through similarity judgments could, in principle, depend on some weakness in our method, such as limited number of participants, speed values, or trials; insufficiently good fitting; extreme criterion values for the same/different responses; and the like. As a matter of fact, $R^{2}$ values indicated that the Gaussian fits were worse than cumulative Gaussian fits in both Experiments 1 and 2 . In order to exclude this possibility, in Experiment 4, we reproduced the comparative and similarity judgment procedure (same number of trials and participants, same test stimuli, and same data analysis) used in the previous three experiments, simulating the effect of attention on the speed of the standard Gabor. We used two values of speed for the standard stimulus, which were roughly equal to the average PSEs obtained in the comparative judgment conditions in Experiments 1 and 2, for the standard-cued and test-cued conditions, respectively. If our method is sound, the PSE values obtained in response to the two different standard values will themselves be statistically different. 
Test Cued
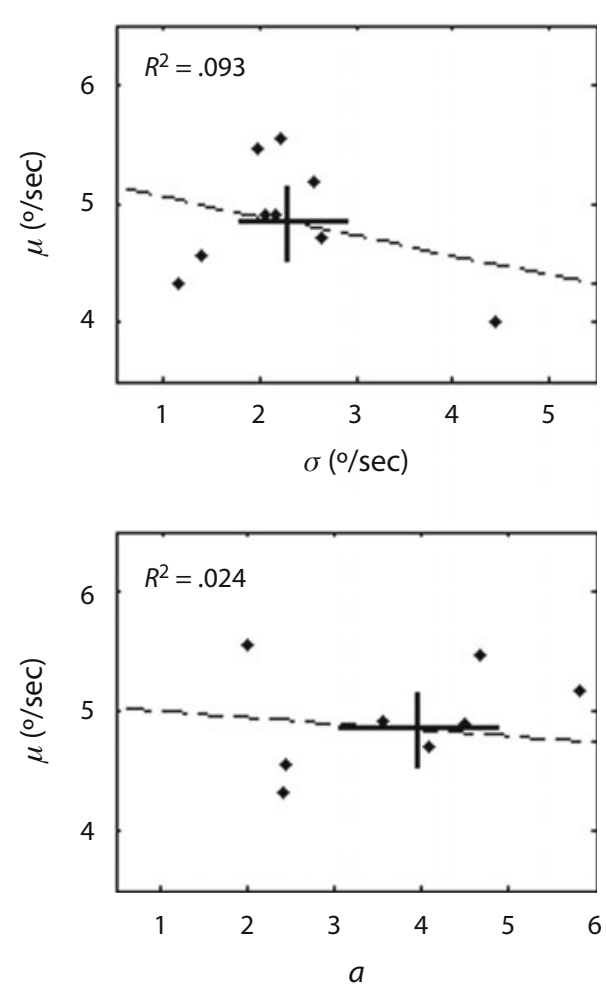

Standard Cued
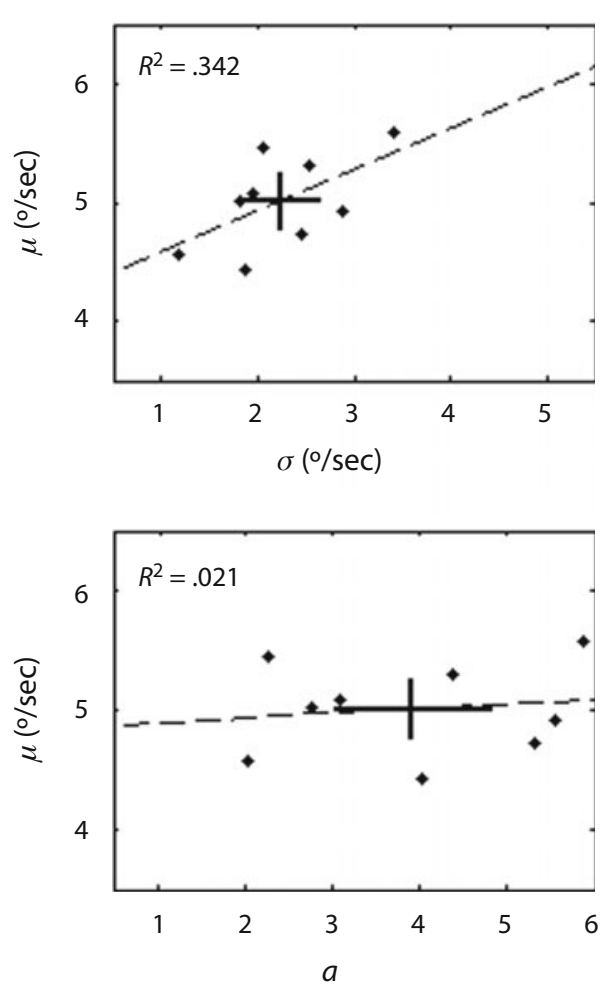

Figure 7. Relationship between fit parameters in Experiment 3. Each point represents a participant and a given condition. The dashed line represents the regression line; the $R^{2}$ value is relative to the linear regression between the two variables. No significant linear relationship was observed in any condition. Error bars indicate $95 \%$ of the distribution of the mean computed from 10,000 bootstrap samples obtained by random resampling with replacement. The coordinates of the crossing point of the two error bars are the between-participants means of the two parameters.

\section{Method}

Participants. Eleven students from the University of Trento (mean age, 26.6 years; 6 of them female) volunteered for participation in Experiment 4 in exchange for course credits or $€ 6$. All of the participants were naive as to the aim of the study and reported normal or corrected-to-normal visual acuity. One of the participants had taken part in Experiment 1, and another had taken part in Experiment 2. All of the participants gave their informed consent, and the experiment was conducted in accordance with the Declaration of Helsinki.

Stimuli and Procedure. The stimuli and procedure were the same as those in Experiments 1-3, with the exception that the cue was not presented and that, at the end of the trials, the participants were asked either to report which Gabor moved faster or to report whether the two Gabors moved at the same speed. The comparative judgment and the similarity judgment tasks were run in separate sessions, which were obtained from the same participants on different days and lasted approximately $45 \mathrm{~min}$ each. The order of the two sessions was counterbalanced between participants, as was the mapping of same and different responses to the up and down arrow keys.

The test Gabors had the same speed values as those in Experiments $1-3$. The standard Gabor moved at a speed of $4.05^{\circ} / \mathrm{sec}$ and $4.69^{\circ} / \mathrm{sec}$ in the low- and high-speed conditions, respectively. Those values are the closest that we could get with our apparatus to the average PSE values in Experiments 1 and 2 in the test- and standardcued conditions, respectively.

Design and Data analysis. The design and data analysis were the same as those in Experiments 1 and 2, with the exception that the standard speed condition took the place of the cuing condition. Since the two types of judgments were collected in different sessions, the total number of trials was twice that in Experiments 1-3 (592 trials in each session).

\section{Results and Discussion}

One participant was discarded from the analysis because he had a poor fit to at least one comparative judgment curve. The average $R^{2}$ of the single participant fits was .97 in the case of the cumulative Gaussian curves and .93 in the case of the Gaussian curves.

The results of the comparative judgments are depicted in Figure 8A. The curve for the slow-speed standard condition was clearly shifted to the left, indicating that the standard was matched to smaller values of the test Gabor, as compared with the one for the high-speed standard condition. The mean value of the PSE for the highspeed standard condition was $4.87^{\circ} / \mathrm{sec} \pm 0.07$ (betweenparticipants $S E M$ ), whereas it was $4.24 \%$ sec \pm 0.06 for the low-speed standard condition. The difference between the two PSE values, as shown by the paired $t$ test, was significant $[t(9)=13.91, p<.001]$.

Cuing did not have a significant effect on the $\sigma$ parameter either (both $t \mathrm{~s}<1, p \mathrm{~s} \geq .585$ ). Individual values of PSE and $\sigma$ are depicted in Figure 9. No significant linear 
A

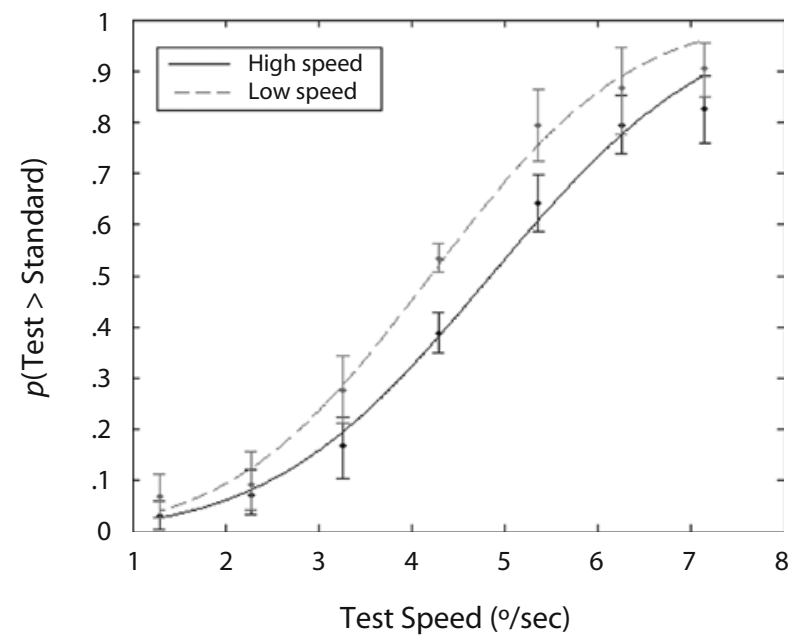

B

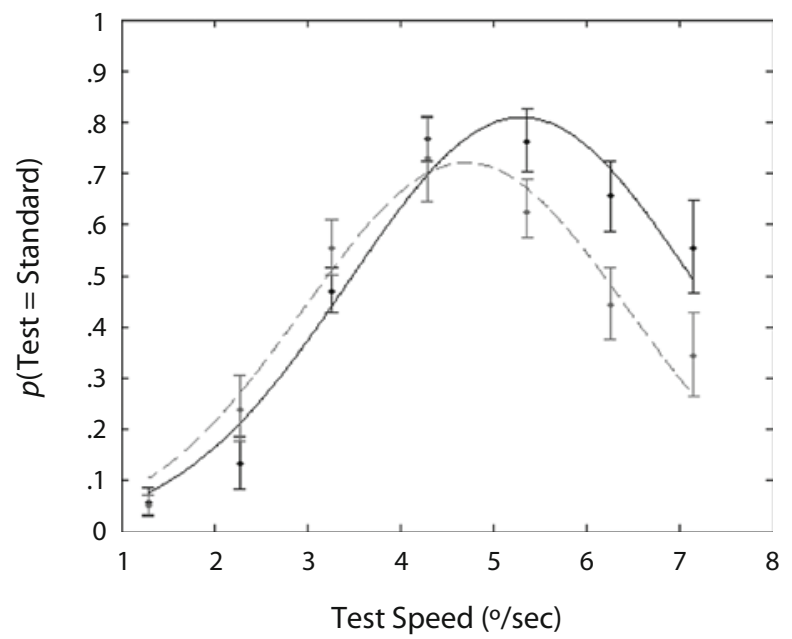

Figure 8. Comparative judgment (A) and similarity judgment (B) results in Experiment 4, for the high-speed standard and lowspeed standard conditions. A cumulative Gaussian curve (A) and a Gaussian curve (B) were fitted to the mean data from the comparative and the similarity measures, respectively. Error bars indicate $95 \%$ of the distribution of the mean computed from 10,000 bootstrap samples obtained by random resampling with replacement.

relationship was observed between $\sigma$ and the PSE in any condition.

The results of the similarity judgments are depicted in Figure 8B, where it is evident that the curves were different. The mean value of the PSE for the high-speed standard condition was $5.33^{\circ} / \mathrm{sec} \pm 0.13$, whereas it was $4.74 \% \mathrm{sec}$ \pm 0.11 for the low-speed standard condition. The difference between the two PSE values was significant $[t(9)=$ 5.47, $p<.001]$. This result was highly reliable; in fact, any combination of 6 or more participants and $81.7 \%$ of all combinations of 5 participants yielded a $p<.05$ significant difference.

It is also evident that the PSE values estimated by the similarity judgment were, in general, higher than the ones produced by the comparative judgments. In order to substantiate this impression and to directly compare the two measures, individual PSE values were further analyzed with a two-way repeated measures ANOVA with standard speed (high vs. low) and judgment type (similarity vs. comparative) as factors. The effects of judgment type $[F(1,9)=81.45, p<.001]$ and standard speed $[F(1,9)=$ 28.03, $p<.001]$ were significant, but their interaction was not $[F(1,9)=0.16, p=.698]$.

Cuing also had a significant effect on the $\sigma$ and $a$ parameters. The value of $\sigma$ was $1.88^{\circ} / \mathrm{sec} \pm 0.11$ in the high-speed standard condition and $1.75 \% \mathrm{sec} \pm 0.10$ in the low-speed standard condition $[t(9)=3.09, p<.013]$. The value of $a$ was $3.86 \pm 0.26$ in the high-speed standard condition and $3.20 \pm 0.19$ in the low-speed standard condition $[t(9)=5.78, p<.001]$. Individual values of PSE, $\sigma$, and $a$ are depicted in Figure 7. A significant (positive) linear relationship was observed between both the $\sigma$ and $a$ parameters and the PSE in both the high-speed standard and the low-speed standard conditions.
The present findings indicate that when the speed of the standard Gabor was shifted by a speed amount comparable to the shift allegedly produced by attention, an effect was reliably produced in the PSE values assessed with similarity judgments. The amplitude of this effect was not different from the one that was observed with comparative judgments. This indicates that, had the exogenous cues in Experiments 1-3 produced a genuine perceptual effect, this would have been detected by our experimental procedure in both comparative and similarity judgments.

It is interesting to note the main effect the type of judgment had on PSE values. The fact that PSEs assessed through similarity judgments were higher than the physical speed of the standard stimulus suggests that higher speed test stimuli tended to appear more similar to the standard speed stimulus, as compared with lower speed test stimuli. The same trend was also quite evident in the data from Experiment 3.

The results of Experiment 4 indicate that increasing the speed of the standard stimulus produces a reliable effect on all three parameters of the Gaussian curve. In particular, both the spread and the amplitude of the functions are increased by cuing, along with the mean. This is coherent with the observation that higher speed stimuli appear more similar to each other than do lower speed stimuli. Given this asymmetry, the participants were more likely to report higher speed stimuli as same, as shown by the increase in the $a$ parameter, and over a wider range, as shown by the increase in the $\sigma$ parameter.

The same asymmetry might be at the basis of the (positive) relation between the $\sigma$ and $a$ parameters and PSE, which we found in most of the conditions in Experiment 4, assuming that the relationship between perceived speed and perceived similarity holds even between par- 
High-Speed Standard
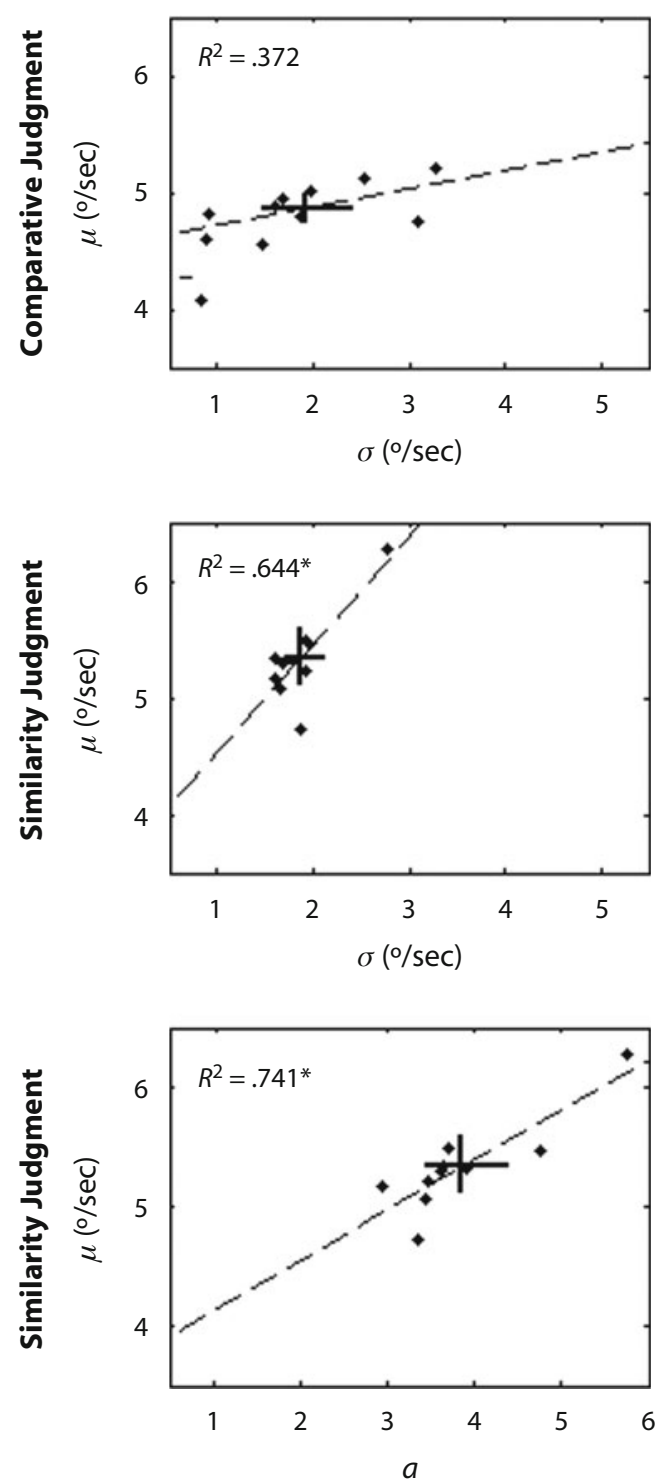

Low-Speed Standard
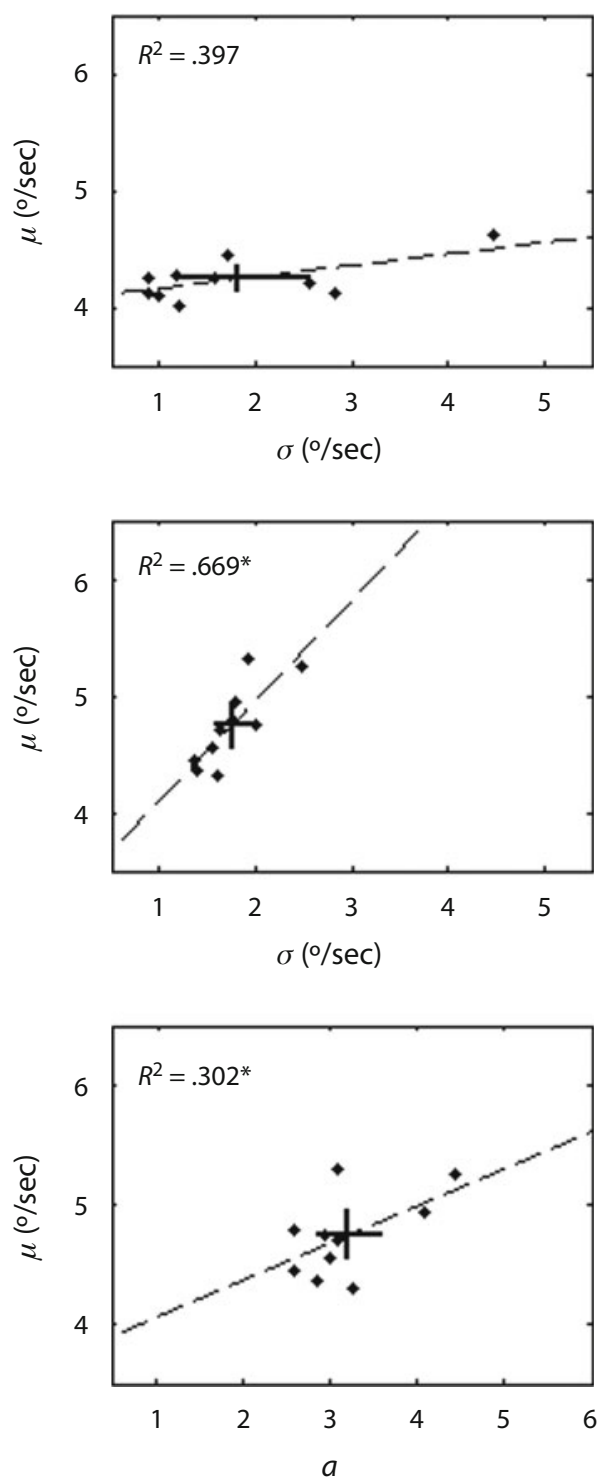

Figure 9. Relationship between fit parameters in Experiment 4. Each point represents a participant and a given condition. The dashed line represents the regression line; the $\boldsymbol{R}^{2}$ value is relative to the linear regression between the two variables. Asterisks indicate a significant $(p<.05)$ linear relationship. Error bars indicate $95 \%$ of the distribution of the mean computed from 10,000 bootstrap samples obtained by random resampling with replacement. The coordinates of the crossing point of the two error bars are the between-participants means of the two parameters.

ticipants. Such a hypothesis, however, must be taken with care, given that similar relations between parameter values were not observed in the previous experiments (with the exception of the $a$ parameter in the standard-cued condition in Experiment 1).

This asymmetry was less evident in comparative judgments, which might point to a dissociation between proper appearance and performance, as assessed by forced choices. This is an effect that might deserve to be studied further, but it has no direct implication for the main result of the present experiment: Similarity judgments are a reli- able procedure for detecting perceptual differences when present.

\section{GENERAL DISCUSSION}

In three experiments, we constantly found no evidence for an attentional enhancement of perceived speed of moving stimuli as indexed by similarity judgments, whereas a strong tendency to report the cued stimulus as moving faster was observed when comparative judgments were used in Experiments 1 and 2. 
By reversing the order of the tasks between Experiments 1 and 2 and by asking the participants to perform only the similarity judgment in Experiment 3, we made sure that the lack of attentional modulation on similarity judgments observed in Experiment 1 was not due to a degradation of the stimuli's sensory representation before the similarity judgment or to the commitment of more resources to the comparative judgment task.

In the model proposed by Schneider and Komlos (2008), this pattern of results is explained in terms of decision biases, rather than perceptual modulations. In their model, an earlier perceptual stage (common to comparative and similarity judgments) is followed by a decision stage, which, in the case of comparative judgments, is prone to biases induced by the attentional prioritization of one of the two stimuli.

Both the model proposed by Schneider and Komlos (2008) and the one proposed by Fuller, Rodriguez, and Carrasco (2008) are formulated in the signal detection theory framework. In both models, the signal intensities produced by the two stimuli are evaluated, leading to a difference signal. This difference signal is then compared with a criterion in order to make a decision. We consider all the stages that lead to the computation of the difference signal to be perceptual and the criterion setting and evaluation to be decisional. In the model proposed by Fuller et al., attention produces a change in the sensory signal and, consequently, in the distribution of the difference signal, whereas in the one in Schneider and Komlos, attention affects the setting of the criterion. However, if attention modified the difference signal intensity, as is postulated by Fuller et al., any kind of judgment based on its relative or absolute value should be likewise affected, which is not what we found. Moreover, it is clear that a change in the intensity of the signal taking place before it is compared with the criterion is equivalent to a change occurring at any earlier stage - for instance, in the stimulus itself. The fact that a physical change and cuing do not have the same effect on similarity judgments is not straightforward if one assumes that the effects of attention intervene before the signal evaluation.

Although being less prone to cue-induced decision or response biases, since the participant is not asked to choose and respond to either the cued or the uncued stimulus, similarity judgments, like any task involving categorization, are not immune from decision biases in general. A number of experimental variables, such as instructions (Ratcliff \& Hacker, 1981) and stimulus probabilities (Coltheart \& Curthoys, 1968), can influence the overall rate of same responses in a similarity judgment task. However, simply making the criterion more liberal in order to increase the overall rate of same responses will not change the estimated value of PSE in any systematic way, except for an increase in the variability of the estimate when the rates are too extreme and the resulting curves are too flat to be fit reliably. To avoid this potential problem, we discarded the participants who did not provide reliable estimates of the PSE. The PSE estimates obtained by similarity judgments are less prone to cue biases in the decision stage than are those obtained by comparative judgments, given that the participant is not asked to decide whether the cued stimulus is greater or smaller with respect to the dimension of interest. The decision is made in terms of similarity or difference, and a general tendency to respond more or less likely same would not affect the central tendency measure of the psychometric curve.

On the other hand, we must observe that the similarity judgment method for estimating the PSE is less effective than the comparative judgment method. It is clear from the data in the first two experiments that the fits of the psychometric curves were poorer in the former case, and a higher number of levels of the standard stimulus speed are needed in order to characterize a Gaussian function, as compared with a cumulative Gaussian one. In fact, the inclusion of the amplitude parameter in the Gaussian model could by itself increase the variability of the other two parameters (e.g., McKee, Klein, \& Teller, 1985). Furthermore, there is evidence of the fact that the similarity judgments might be harder to perform. Fetterman, Dreyfus, and Stubbs (1996) demonstrated that participants are less precise in similarity judgments of duration than in comparative judgments of duration and that similarity judgment rules are less easy to learn from informative feedback provided to the participants after each choice.

Another issue that might undermine the effectiveness of our method is the fact that the criterion used by the participants when answering same/different questions depends on the proportion of same trials (e.g., Coltheart \& Curthoys, 1968). In Experiments 1-3, the proportion of trials on which the test and the standard stimulus had the same speed was 1/7. In Experiment 4, there were no same trials at all, and theoretically, the same holds for the first three experiments if one assumes that cuing had a perceptual effect on attention. In fact, an ideal participant would never have provided the same response, making PSE estimation impossible. However, in most of the cases, the participants produced a sufficient number of same responses, and their proportion decreased when the speed difference between the two stimuli increased, making PSE estimation possible.

It should be noted, however, that the choice of using similarity judgments was justified not by their superiority in terms of effectiveness in PSE estimation, but by their lesser proneness to decision biases induced by the cue. This is due to the fact that similarity judgments do not require the participant to decide which stimulus has higher contrast, but to decide whether they have the same speed. For the purpose of the present study, it was crucial to show that similarity judgments were just sensitive enough to detect PSE difference of the magnitude we were looking for, and this is precisely what we found in Experiment 4 . The results of Experiment 4 indicate that the numerous weaknesses of the similarity judgment method do not explain the different effect of cuing between similarity and comparative judgments. We suggest a decision bias limited to the case of comparative judgments as a possible explanation.

The practice of letting participants provide a same response, instead of the comparative judgment, is rooted in the practice of psychophysics since Fechner (1966), who would allow participants to provide a "doubtful" answer 
in his method of right and wrong cases, and the perception of sameness is at the basis of the method of limits. The use of the same category in psychophysical judgments was later at the basis of the calculation of the interval of uncertainty, defined as the difference between the thresholds calculated separately for the lower and higher responses (Urban, 1910). The use of same responses in discrimination tasks has been criticized because of the fact that the total amount of same responses can differ strongly between participants and can be influenced by task instructions (e.g., Angell, 1907; Fernberger, 1930). Unforced choice tasks are, however, considered more comfortable to use with untrained participants, as compared with forced choice tasks (Kaernbach, 2001), and are more easily performed by primates, as compared with confidence ratings (Smith, Shields, Schull, \& Washburn, 1997). Moreover, it has been demonstrated that the inclusion of the uncertain response class does not by itself produce more unreliable threshold estimates (Kaernbach, 2001).

The practice of constructing psychometric curves for similarity judgments is much less common (see Brown, 1910 , for an early example). The fact that fitting bellshaped curves requires more complex models than in the case of sigmoid-like functions, together with the problem of variable interparticipant criteria, has limited the use of similarity judgments for the purpose of PSE estimation. However, the related issue of assessing the point of subjective simultaneity in the field of arrival time judgments has sometimes been addressed with the equivalent of similarity judgments - that is, simultaneity judgments.

The problem of establishing whether attention biases the decision stage or the perceptual stage in a forced choice task regarding a visual attribute is similar to the problem of establishing whether attention produces a genuine prior entry in the domain of time perception. Simultaneity judgments have been proposed as a bias-free tool to assess this question (Schneider \& Bavelier, 2003). A number of studies have shown that the point of subjective simultaneity, as assessed by simultaneity judgments, is shifted by attention (Santangelo \& Spence, 2008; Van der Burg, Olivers, Bronkhorst, \& Theeuwes, 2008; Zampini, Shore, $\&$ Spence, 2005), indicating that the prior entry effect is perceptual in nature. Both Santangelo and Spence and Van der Burg et al. found smaller attentional effects in the simultaneity judgment PSEs, as compared with time order judgments, and interpreted this as an indication that time order judgments are partially contaminated by decision/ response biases.

In general, studies on the effects of attention on simultaneity judgments have demonstrated that if attention really modifies a perceptual attribute, this can be detected by asking participants to categorize the stimuli in terms of their similarity along the continuum of interest (in that case, the arrival time) and by estimating the point at which the probability of responding same is higher (see van Eijk, Kohlrausch, Juola, \& van de Par, 2008, for a comparison of time order and simultaneity judgment tasks).

Although similarity judgments seem to be less effective, because of the need to add one parameter to the model (McKee et al., 1985), or more difficult to learn and perform (Fetterman et al., 1996), whenever two stimuli differ beyond the feature on which the participants have to make a comparison, the results cannot be univocally interpreted in perceptual terms, since the participants' choices might, in fact, be biased by the difference in the irrelevant features. This is particularly true in the case of cuing, which, by definition, enhances the saliency of the stimulus. Comparative judgments can be considered bias free only as long as the design allows one to equate or randomize any aspect of the stimuli other than the dimension of interest. If this is not possible, as in the case in which attentional cuing, position, or sensory modality needs to differ between the stimuli, a similarity judgment task should be preferred.

The results of the present study do not imply that attention does not affect any motion-related signal in the brain, since there is, in fact, evidence to the contrary. For example, attention shifts induced by microstimulation of primate frontal eye fields enhance the motion-induced displacement illusion (Schafer \& Moore, 2007). The fact that this illusory displacement of static motion signals in the direction of motion (De Valois \& De Valois, 1991) is modulated by attention shifts does not necessarily contradict the present results, which indicate solely that the effect of attention on reported speed is not likely to be perceptual in nature. Attention could modify motion signals that are not used for report but may affect some aspects of behavior.

On the basis of the present data and of the data reported by Turatto et al. $(2007,2008)$, we can conclude that the tendency to report the cued stimulus as moving faster in comparative judgments is a genuinely attentive effect, given that it disappears when the cue-target SOA is extended (Turatto et al., 2007, Experiment 2). There is, however, no mandatory link between attentional orienting and comparative enhancement of perceived speed, given that attention oriented by memory cues does not affect comparative speed judgments (Turatto et al., 2008). Furthermore, we are not dealing with a trivial response bias, given that the cuing effect reverses when the response mapping is reversed (Turatto et al., 2007, Experiment 3). However, the fact that no trace of attentional enhancement of perceived speed, as indexed by similarity judgments, was observed in the present study suggests that the enhancement indexed by the comparative judgments arises at a decisional stage.

\section{AUTHOR NOTE}

Correspondence concerning this article should be addressed to M. Valsecchi, Department of General Psychology, Justus-LiebigUniversität Giessen, Otto-Behaghel-Strasse 10F, D-35394 Giessen, Germany (e-mail: matteo.valsecchi@gmail.com).

\section{REFERENCES}

ANGELL, F. (1907). On judgments of "like" in discrimination experiments. American Journal of Psychology, 18, 253-260.

Anton-ErXleben, K., Henrich, C., \& Treue, S. (2007). Attention changes perceived size of moving visual patterns. Journal of Vision, 7(11, Art. 5), 1-9. doi:10.1167/7.11.5

Brown, W. (1910). The judgment of difference. Berkeley: University of California Press. 
Carrasco, M., Fuller, S., \& Ling, S. (2008). Transient attention does increase perceived contrast of suprathreshold stimuli: A reply to Prinzmetal, Long, and Leonhardt (2008). Perception \& Psychophysics, 70, 1151-1164. doi:10.3758/PP.70.7.1151

Carrasco, M., Ling, S., \& Read, S. (2004). Attention alters appearance. Nature Neuroscience, 7, 308-313.

Coltheart, M., \& Curthoys, I. (1968). Short-term recognition memory for pitch: Effect of a priori probability on response times and error rates. Perception \& Psychophysics, 4, 85-89.

De Valois, R. L., \& De Valois, K. K. (1991). Vernier acuity with stationary moving Gabors. Vision Research, 31, 1619-1626.

FeCHNER, G. (1966). Elements of psychophysics (H. E. Adler, Trans.). New York: Holt, Rinehart \& Winston.

Fernberger, S. W. (1930). The use of equality judgments in psychophysical procedures. Psychological Review, 37, 107-112.

Fetterman, J. G., Dreyfus, L. R., \& Stubbs, D. A. (1996). Judging relative duration: The role of rule and instructional variables. Journal of Experimental Psychology: Animal Behavior Processes, 22, 350-361.

Fuller, S., \& Carrasco, M. (2006). Exogenous attention and color perception: Performance and appearance of saturation and hue. Vision Research, 46, 4032-4047. doi:10.1016/j.visres.2006.07.014

Fuller, S., Rodriguez, R. Z., \& Carrasco, M. (2008). Apparent contrast differs across the vertical meridian: Visual and attentional factors. Journal of Vision, 8(1, Art. 16), 1-16. doi:10.1167/8.1.16

Gobell, J., \& CARrasco, M. (2005). Attention alters the appearance of spatial frequency and gap size. Psychological Science, 16, 644-651.

KAERNBACH, C. (2001). Adaptive threshold estimation with unforcedchoice tasks. Perception \& Psychophysics, 63, 1377-1388.

Ling, S., \& CARrasco, M. (2007). Transient covert attention does alter appearance: A reply to Schneider (2006). Perception \& Psychophysics, 69, 1051-1058.

LiU, T., Abrams, J., \& CARrasco, M. (2009). Voluntary attention enhances contrast appearance. Psychological Science, 20, 354-362.

Liu, T., Fuller, S., \& Carrasco, M. (2006). Attention alters the appearance of motion coherence. Psychonomic Bulletin \& Review, 13, 1091-1096.

McKee, S. P., Klein, S. A., \& Teller, D. Y. (1985). Statistical properties of forced-choice psychometric functions: Implications of probit analysis. Perception \& Psychophysics, 37, 286-298.

Montagna, B., \& Carrasco, M. (2006). Transient covert attention and the perceived rate of flicker. Journal of Vision, 6, 955-965. doi: $10.1167 / 6.9 .8$

PELLI, D. G. (1997). The VideoToolbox software for visual psychophysics: Transforming numbers into movies. Spatial Vision, 10, 437-442.

Prinzmetal, W., Long, V., \& Leonhardt, J. (2008). Involuntary attention and brightness contrast. Perception \& Psychophysics, 70, 11391150. doi:10.3758/PP.70.7.1139

RATCliff, R., \& Hacker, M. J. (1981). Speed and accuracy of same and different responses in perceptual matching. Perception \& Psychophysics, 30, 303-307.

Santangelo, V., \& Spence, C. (2008). Crossmodal attentional capture in an unspeeded simultaneity judgement task. Visual Cognition, 16, 155-165. doi:10.1080/13506280701453540
Schafer, R. J., \& Moore, T. (2007). Attention governs action in the primate frontal eye field. Neuron, 56, 541-551. doi:10.1016/j .neuron.2007.09.029

SchneIder, K. A. (2006). Does attention alter appearance? Perception \& Psychophysics, 68, 800-814.

Schneider, K. A., \& Bavelier, D. (2003). Components of visual prior entry. Cognitive Psychology, 47, 333-366. doi:10.1016/S0010 $-0285(03) 00035-5$

Schneider, K. A., \& Komlos, M. (2008). Attention biases decisions but does not alter appearance. Journal of Vision, 8(15, Art. 3), 1-10. doi:10 $.1167 / 8.15 .3$

Smith, J. D., Shields, W. E., Schull, J., \& Washburn, D. A. (1997). The uncertain response in humans and animals. Cognition, 62, 75-97.

Turatto, M., Vescovi, M., \& Valsecchi, M. (2007). Attention makes moving objects be perceived to move faster. Vision Research, 47, 166178. doi:10.1016/j.visres.2006.10.002

Turatto, M., Vescovi, M., \& Valsecchi, M. (2008). On altering motion perception via working memory-based attention shifts. Journal of Vision, 8(5, Art. 11), 1-13. doi:10.1167/8.5.11

Urban, F. M. (1910). The method of constant stimuli and its generalizations. Psychological Review, 17, 229-259.

VAn der Burg, E., Olivers, C. N. L., Bronkhorst, A. W., \& TheeuWES, J. (2008). Audiovisual events capture attention: Evidence from temporal order judgments. Journal of Vision, 8(5, Art. 2), 1-10. doi: $10.1167 / 8.5 .2$

van EiJK, R. L. J., Kohlrausch, A., Juola, J. F., \& van de Par, S. (2008). Audiovisual synchrony and temporal order judgments: Effects of experimental method and stimulus type. Perception \& Psychophysics, 70, 955-968. doi:10.3758/PP.70.6.955

Zampini, M., Shore, D. I., \& Spence, C. (2005). Audiovisual prior entry. Neuroscience Letters, 381, 217-222. doi:10.1016/j.neulet .2005 .01 .085

\section{NOTE}

1. The test Gabor speed levels used in this study are roughly linearly spaced and differ from the ones we used in previous experiments $\mathrm{Cu}$ ratto et al., 2007, 2008), where the standard speed levels were logarithmically spaced. In a pilot study, we determined that given that the probability of same responses decreases symmetrically on both the right and left hand of the speed range, using roughly logarithmically spaced speed values $(3.0,3.4,3.8,4.3,5.0,6.1,7.6)$ produced a suboptimal sampling of the left tail of the distribution for the similarity measure. We were able to fit a Gaussian function only to the responses of 6 out of 16 participants (considering acceptable the fits where the $95 \%$ confidence interval for the PSE was smaller than $3.5^{\circ} / \mathrm{sec}$ ). The data from this pilot experiment failed to show any evidence of enhancement of perceived speed by exogenous cues, coherent with the data obtained in the present study.

(Manuscript received April 6, 2009 revision accepted for publication October 19, 2009.) 